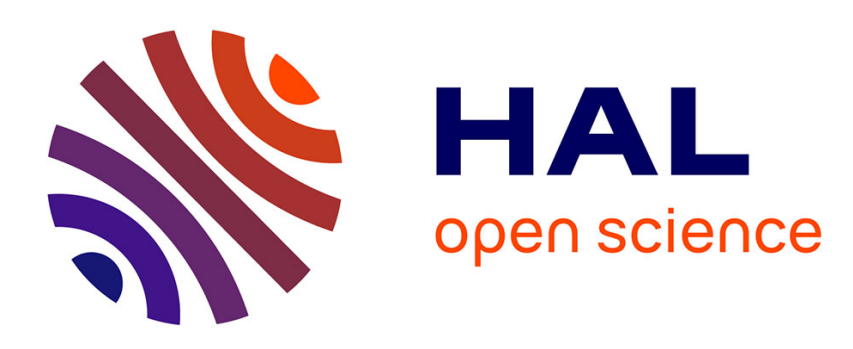

\title{
From a Closed Piecewise Geodesic to a Constriction on a Closed Triangulated Surface
}

Franck Hétroy, Dominique Attali

\section{To cite this version:}

Franck Hétroy, Dominique Attali. From a Closed Piecewise Geodesic to a Constriction on a Closed Triangulated Surface. Pacific Graphics Conference on Computer Graphics and Applications, J. Rokne, Oct 2003, Canmore, Alberta, Canada, France. pp.394-398. inria-00001145

\section{HAL Id: inria-00001145 https://inria.hal.science/inria-00001145}

Submitted on 9 Nov 2016

HAL is a multi-disciplinary open access archive for the deposit and dissemination of scientific research documents, whether they are published or not. The documents may come from teaching and research institutions in France or abroad, or from public or private research centers.
L'archive ouverte pluridisciplinaire HAL, est destinée au dépôt et à la diffusion de documents scientifiques de niveau recherche, publiés ou non, émanant des établissements d'enseignement et de recherche français ou étrangers, des laboratoires publics ou privés. 


\title{
From a closed piecewise geodesic to a constriction on a closed triangulated surface
}

\author{
Franck Hétroy, Dominique Attali \\ LIS Laboratory, INPG, Grenoble, France \\ $\{$ Franck.Hetroy, Dominique.Attali \}@lis.inpg.fr
}

\begin{abstract}
Constrictions on a surface are defined as simple closed curves whose length is locally minimal. In particular, constrictions are periodic geodesics. We use constrictions in order to segment objects. In [4], we proposed an approach based on progressive surface simplification and local geodesic computation. The drawback of this approach is that constrictions are approximated by closed piecewise geodesics which are not necessarily periodic geodesics. In this paper, we compute constrictions starting from the closed piecewise geodesics previously computed and moving them on the surface. We compare the location of the initial closed piecewise geodesics to the location of the constrictions. Finally, we define and compute different types of constrictions on a surface.
\end{abstract}

Keywords: segmentation, triangulated surface, constriction, geodesic, pivot vertex.

\section{Introduction}

In order to handle complex objects, it can be useful to decompose them into several characteristic components, called features. Different definitions of features are possible. For example, we can segment an object into parts that are connected by bottlenecks. The skeleton [1] provides a global shape description [7] that can be used in order to localize the bottlenecks of an object. Such an approach has been used in mathematical morphology [8], for shape decomposition [5]. More recently, the concept of dynamical systems has been proposed to define features of shapes [2].

In this paper, we identify the narrower parts of an object by computing a set of closed curves directly on the boundary of the object. Those curves are called constrictions and are defined as simple closed curves whose length is locally minimal with respect to the Hausdorff distance. In particular, constrictions are periodic geodesics. In
[4], we proposed a method to construct closed piecewise geodesics on triangulated surfaces. A drawback of this approach is that the curves are not necessarily constrictions. The goal of this paper is to compute constrictions starting from these curves. The idea is to move the curves on the surface until they become periodic geodesics. We investigate the efficiency of the algorithm proposed in [4], that is to say we study the distance between a computed closed piecewise geodesic and the corresponding constriction.

We first recall some basic definitions and properties concerning constrictions (Section 2). We briefly recall our method to compute closed piecewise geodesics in Section 3. We explain our "sliding" algorithm to compute constrictions starting from the closed piecewise geodesics (Section 4). In Section 5 we define two kinds of constrictions and we propose an algorithm to distinguish between them. Section 6 is devoted to the comparison between constrictions and computed closed piecewise geodesics. We conclude in Section 7 .

\section{Definitions}

Let $\mathcal{P}$ be a 2 -manifold embedded in $\mathbb{R}^{3}$, and $\mathcal{C}$ the set of simple, closed curves on $\mathcal{P}$. We denote by $L(\alpha)$ the length of a simple closed curve $\alpha \in \mathcal{C}$, and $d(p, q)$ the geodesic distance between two points $p$ and $q$ of $\mathcal{P}$. The Hausdorff distance between two subsets (in our case, two curves) $\alpha$ and $\beta$ of $\mathcal{P}$ is

$$
d_{H}(\alpha, \beta)=\max \left(\sup _{x \in \alpha}\left(\inf _{y \in \beta} d(x, y)\right), \sup _{y \in \beta}\left(\inf _{x \in \alpha} d(x, y)\right)\right) .
$$

The open ball of radius $\varepsilon$ and centered at $\alpha$ on $\mathcal{C}$ is defined as $\mathcal{B}(\alpha, \varepsilon)=\left\{\beta \in \mathcal{C}, d_{H}(\alpha, \beta)<\varepsilon\right\}$.

We call closed geodesic starting from $p$ a geodesic curve on $\mathcal{P}$ between the point $p$ and itself. We call periodic geodesic a closed curve $\gamma$ such that $\forall p \in \gamma, \gamma$ is a closed geodesic starting from $p$. 
Definition $1 \alpha \in \mathcal{C}$ is a constriction if there exists $\varepsilon>0$ such that $\forall \beta \in \mathcal{B}(\alpha, \varepsilon), L(\alpha) \leq L(\beta)$.

Thus constrictions on $\mathcal{P}$ are periodic geodesics on $\mathcal{P}$. We now assume that the surface $\mathcal{P}$ is triangulated.

Definition $2 A$ pivot vertex of a curve $\alpha \in \mathcal{C}$ is a vertex of $\mathcal{P}$ through which $\alpha$ goes.

Definition $3 A$ sequence of faces on $\mathcal{P}$ is a list of faces $\left(F_{1}, F_{2}, \ldots, F_{n}\right)$ such that $\forall i \in\{1, \ldots, n-1\}, F_{i}$ is adjacent to $F_{i+1}$.

Definition 4 Let $\alpha$ be a geodesic path on a $\mathcal{P}$. Let $p \in \alpha$ and $T(p)$ be the faces of $\mathcal{P}$ containing $p$. Let $r$ be a strictly positive real number such that the geodesic circle $C(p, r)$ centered at $p$ with radius $r$ is contained in $T(p)$ and $\alpha$ cuts $C(p, r)$ in two connected components of length $l_{1}$ and $l_{2}$. The angle made by $\alpha$ at point $p$ is defined as the minimum of $l_{1} / r$ and $l_{2} / r$.

We now give a characterization of constrictions, the proof of which can be found in [4]. This characterization is used to identify which curves are constrictions.

Proposition 5 (Characterization of constrictions) Let $\alpha$ be a simple, closed curve on $\mathcal{P}$. Let $p_{1}, \ldots, p_{n}$ be the pivot vertices of $\alpha$. If $\alpha$ unfolds to a straight line segment on $\mathcal{P}$ between any two successive vertices $p_{i}$ and $p_{i+1}$ and the angle of $\alpha$ at each $p_{i}$ is greater than, or equal to, $\pi$, then $\alpha$ is a constriction.

\section{Computing closed piecewise geodesics}

We now briefly describe the algorithm proposed in [4], which computes closed piecewise geodesics. This algorithm works in two steps.

First, it progressively simplifies the surface $\mathcal{P}^{0}$, using the edge collapse operator and maintaining the topology of the surface. Let us denote $\mathcal{P}^{k}$ the simplified surface after $k$ edge collapses. On each surface $\mathcal{P}^{k}$, the algorithm detects seed curves, which are curves made of three edges $a b, b c$ and $c a$ such that the triangle $a b c$ is not a triangle of $\mathcal{P}^{k}$. The simplification process stops when no edge collapse is possible without modifying the topology of the surface.

Second, the algorithm goes back to the initial surface $\mathcal{P}^{0}$, computing closed piecewise geodesics on each $\mathcal{P}^{k}$. Let us assume we have detected a seed curve $\alpha_{n}$ on $\mathcal{P}^{n}$. The algorithm build a sequence of closed piecewise geodesic $\alpha_{n-1}, \ldots, \alpha_{0}$ on the sequence of simplified surfaces $\mathcal{P}^{n-1}, \ldots, \mathcal{P}^{0}$. In order to define $\alpha_{i}, \alpha_{i+1}$ is modified between two pivot vertices, in case the surface is altered between these two vertices: a geodesic curve is computed between them.
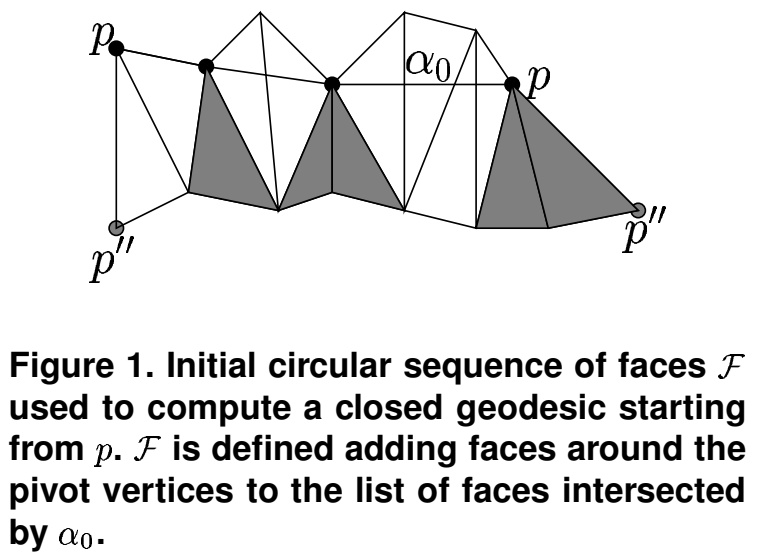

\section{Computing constrictions}

\subsection{Computing closed geodesics}

The curves computed with the algorithm of [4] are closed piecewise geodesics, but may not be constrictions. According to Proposition 5, a closed piecewise geodesic $\alpha_{0}$ is a constriction if, and only if, the angle made by $\alpha_{0}$ at each pivot vertex is at least $\pi$. Instead of checking the angle at every pivot vertices of $\alpha_{0}$, we prefer to compute a closed geodesic starting from a pivot vertex $p$. In order to check if the curve $\alpha_{0}$ is a periodic geodesic, we only have to check the angle at $p$, since the angle made by a geodesic curve at a pivot vertex is at least $\pi$.

Let $\alpha_{0}$ be a closed piecewise geodesic. Let $p$ be a pivot vertex of $\alpha_{0}$. We compute a geodesic starting from $p$ using Pham-Trong's algorithm [6]. This algorithm needs an initial sequence of faces $\mathcal{F}$. To define $\mathcal{F}$, we start from the list of faces intersected by $\alpha_{0}$. We add faces around the pivot vertices in order to get a sequence of faces. We also add faces around $p$ at the end of the list in order to get a circular sequence, see Figure 1. Then, we can compute a geodesic between $p$ and $p$, using [6] and starting from the circular sequence of faces $\mathcal{F}$. Figure 4 (a) shows the closed piecewise geodesic $\alpha_{0}$ together with the initial sequence of faces and the starting vertex $p$. Figure 4 (b) shows the closed geodesic $\alpha_{0}^{\prime}$ starting from $p$ which has been computed.

\subsection{Checking the angle at the starting vertex}

Once we have computed a closed geodesic $\alpha_{0}^{\prime}$ starting from a vertex $p$, we must check if the angle made by $\alpha_{0}^{\prime}$ at $p$ is greater than, or equal to, $\pi$. If this is the case, $\alpha_{0}^{\prime}$ is a constriction and the algorithm stops. Otherwise, $\alpha_{0}^{\prime}$ is not a constriction: we compute a new closed geodesic $\alpha_{0}^{\prime \prime}$, starting from another pivot vertex $p^{\prime}$ of $\alpha_{0}^{\prime}$. The new initial cir- 
cular sequence of faces is defined using the list of faces intersected by $\alpha_{0}^{\prime}$, and adding faces as described in Section 4.1. In Figure 4 (b), the angle made by $\alpha_{0}^{\prime}$ at $p$ is lower than $\pi$. The new closed geodesic $\alpha_{0}^{\prime \prime}$ starting from another pivot vertex $p^{\prime}$ is shown in Figure 4 (c).

\subsection{Evolution of the starting vertex}

Assume $p$ was the only pivot vertex of the closed geodesic $\alpha_{0}^{\prime}$. In this case, the new closed geodesic will start from a vertex $p^{\prime \prime}$ through which $\alpha_{0}^{\prime}$ does not go. $p^{\prime \prime}$ is chosen as the other endpoint of the common edge of the first and last faces of the previous circular sequence, see Figure 1. This solution, proposed by Szafran [9], allows the curve to "slide" along the surface. We continue to compute closed geodesics until the angle made by the curve at its starting vertex is greater than, or equal to, $\pi$.

In order to avoid an endless loop, we must ensure at each step that the starting vertex is different from previous ones. To do so, we store the vertices already visited in a list. If no new starting vertex can be chosen, the algorithm stops and points out that no constriction has been computed. Figure 4 (d) shows a constriction $\alpha_{0}^{\prime \prime \prime}$ constructed starting from a neighbouring vertex $p^{\prime \prime}$ of the only pivot vertex $p^{\prime}$ of the closed geodesic $\alpha_{0}^{\prime \prime}$.

\section{First and second type constrictions}

Let $O$ be an object, whose boundary $\mathcal{P}$ is a triangulated 2-manifold embedded in $\mathbb{R}^{3}$. Constrictions are intrinsic to the surface $\mathcal{P}$, since Definition 1 does not depend on the underlying object $O$. Thus, not all computed constrictions correspond to the segmentation of the object. We propose here a method to detect these constrictions, which will be called first type constrictions.

\subsection{Definitions}

Let $\alpha$ be a constriction on $\mathcal{P}$. Let $\operatorname{hull}(\alpha) \subset \mathbb{R}^{3}$ be the convex hull of $\alpha$. If hull $(\alpha)$ divides $O$ into two parts, $\alpha$ is called a first type constriction. If hull $(\alpha)$ divides $\mathbb{R}^{3} \backslash O$ into two parts, $\alpha$ is called a second type constriction. Note that a constriction can be neither a first nor a second type constriction, as illustrated in Figure 2.

\subsection{Detection}

We propose a method to detect if a constriction is a first or a second type constriction. The idea behind this method is the following. Let $O=O^{0}$ be an object and $\mathcal{P}=\mathcal{P}^{0}$ its boundary. Let $\alpha$ be a constriction on $\mathcal{P}^{0} . \forall 1 \leq k \leq n$, we note $\mathcal{P}^{k}$ the simplified surface

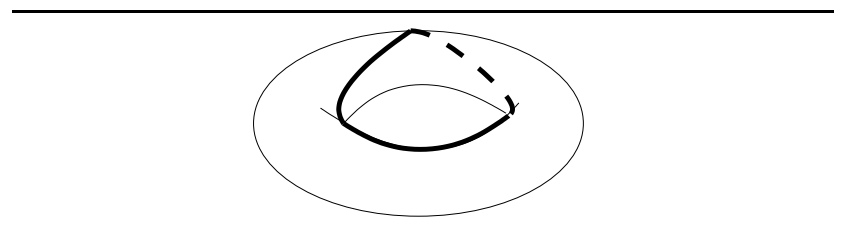

Figure 2. This constriction is neither a first nor a second type constriction.

used to construct $\alpha$ with the algorithm of [4], $O^{k}$ the object whose boundary is $\mathcal{P}^{k}$, and $\alpha_{k}$ the closed piecewise geodesic on $\mathcal{P}^{k}$ constructed with the algorithm of [4]. If the convex hull of $\alpha$ divides the object $O$, it should be the same for all the piecewise geodesics $\alpha_{k}$ with respect to their corresponding objects $O^{k}$. In particular, the convex hull of the seed curve $\alpha_{n}$ which leads to $\alpha$ is likely to divide the object $O^{n}$ bounded by the simplified surface $\mathcal{P}^{n}$. Remember that a seed curve consists of three edges $a b, b c$ and $c a$ such that the triangle $a b c$ is not a triangle of the surface. Thus, the convex hull of such a seed curve is the triangle $a b c$. As a consequence, $\alpha$ should be a first type constriction if the convex hull of $\alpha_{n}$ divides the object $O^{n}$ into two parts, and $\alpha$ should be a second type constriction if the convex hull of $\alpha_{n}$ divides $\mathbb{R}^{3} \backslash O^{n}$ into two parts.

To detect if a triangle $a b c$ is inside the object $O^{n}$, we first compute the intersection of this triangle with all triangles of the simplified surface $\mathcal{P}^{n}$. If this intersection is not empty, the constriction is neither a first type nor a second type constriction. Then, we take a random half-ray $\delta$ starting from the barycenter $d$ of the three points $a, b$ and $c$. If the number of intersections between $\delta$ and the surface $\mathcal{P}^{n}$ is odd, then $a b c$ is inside the object, and the constriction is a first type constriction; else $a b c$ is outside the object, and the constriction is a second type constriction. See Figure 3 for an example.

The main advantage of this algorithm is that we do not need to reconstruct the constriction, not even the closed piecewise geodesic on the initial surface, to determine if we have a first type or a second type constriction. Using this algorithm just after the first step of [4] (the progressive surface simplification process), we can stop if the triangle $a b c$ corresponding to the seed curve $(a b c)$ is outside the object $O^{n}$ bounded by the surface $\mathcal{P}^{n}$.

\section{Results and discussion}

Figures 5 and 6 show the location of constrictions computed on some models, compared with the loca- 

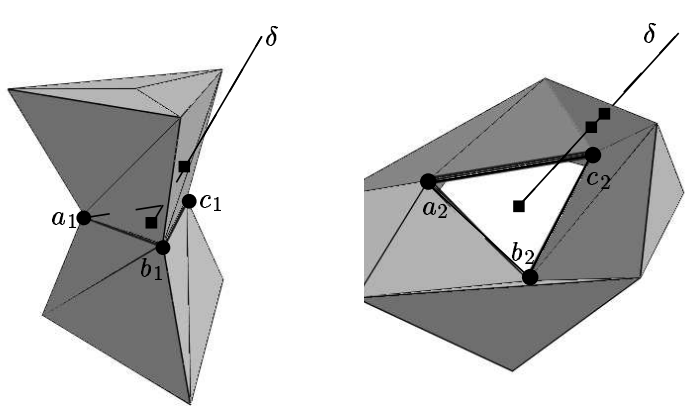

Figure 3. The left seed curve $a_{1} b_{1} c_{1}$ corresponds to a first type constriction. The right seed curve $a_{2} b_{2} c_{2}$ corresponds to a second type constriction. The number of intersections between the random half-ray $\delta$ and the surface is 1 in the first case and 2 in the second case.

tion of closed piecewise geodesics computed with [4]. We can see on these models that the longest closed piecewise geodesics (compared with the diameter of the surface) are often very close to the corresponding constrictions, see e.g. Figure 5 (b) or the legs of the dinosaur in Figure 6. On the contrary, the smallest piecewise geodesics are significantly moved during the "sliding" process, see the legs of the horse in Figure 5 or the neck of the dinosaur. We have noticed that these constrictions go through only one or two pivot vertices, whereas longest constrictions often go through at least three pivots. We also observe that constrictions close to other constrictions, or even constrictions sharing a vertex (Figure 5 (d)), can be computed.

We have tested several simplification methods using the edge collapse operator, such as Garland and Heckbert's [3], or a method which collapses at each step the shortest edge. The resulting vertex is placed according to the heuristic of [3]. Results on the horse model are shown in Figure 7. We see that the number of constructed constrictions and their location highly depends on the simplification algorithm.

\section{Conclusion}

We have proposed an algorithm to turn closed piecewise geodesics into constrictions, based on the construction of a closed geodesic and the computation of the angle made by this curve at its starting vertex. We have also proposed a method to select constrictions which cut the object bounded by the surface. We have compared the location of the closed piecewise geodesics computed by [4] with the location of corresponding constrictions. Long closed piecewise geodesics are very close to constrictions, while the smallest ones, which go through only one or two pivot vertices, need to be slided in order to become constrictions.

Our method may not detect all constrictions on noisy surfaces. This can be of great interest in order to segment the surfaces, since on such surfaces the number of constrictions is theoretically very high; but this can also be a drawback. Moreover, in order to decompose the surface in "homogeneous" components, we need to find a criterion, based for example on the distance between constrictions, to select useful constrictions. Detected constrictions highly depend on the choosen simplification algorithm, which must preserve as much as possible the shape of the surface. A theoretical study of the simplification step should be conducted, in order to keep constrictions and not to create new ones. Finally, the computation time should be studied.

\section{Acknowledgements}

The authors thank Nicolas Szafran and Valérie PhamTrong for explaining their works and for useful and interesting comments about this work.

\section{References}

[1] H. Blum. A transformation for extracting new descriptors of shape. Models for the Perception of Speech and Visual Form, pages 362-380, 1967.

[2] T. Dey, J. Giesen, and S. Goswami. Shape segmentation and matching with flow discretization. In Proc. of Workshop on Algorithms and Data Structures, July 2003.

[3] M. Garland and P. Heckbert. Surface simplification using quadric error metrics. In ACM Computer Graphics (Proc. of SIGGRAPH'97), volume 31, pages 209-216, August 1997.

[4] F. Hétroy and D. Attali. Detection of constrictions on closed polyhedral surfaces. In Data Visualization 2003, Visualization Symposium Proc., pages 67-74, May 2003.

[5] F. Leymarie and B. Kimia. The shock scaffold for representing 3d shape. In Proc. 4th Internat. Workshop on Visual Form (IWVF), LNCS 2059, pages 216-229, May 2001.

[6] V. Pham-Trong, L. Biard, and N. Szafran. Pseudo-geodesics on three-dimensional surfaces and pseudo-geodesic meshes. Numerical Algorithms, 26(4):305-315, April 2001.

[7] G. SannitiDiBaja and S. Svensson. Detecting centres of maximal geodesic dics on the distance transform of surfaces in $3 \mathrm{~d}$ images. In Proc. of Discrete Geometry for Computer Imagery (DGCI), LNCS 1953, pages 443-452, December 2000.

[8] J.-P. Serra. Image Analysis and Mathematical Morphology, volume 1. Academic Press, 1982.

[9] N. Szafran. Chemins et boucles géodésiques sur des polyèdres [geodesic paths and loops on polyhedra]. In Journées de Géométrie Algorithmique, October 2002. 


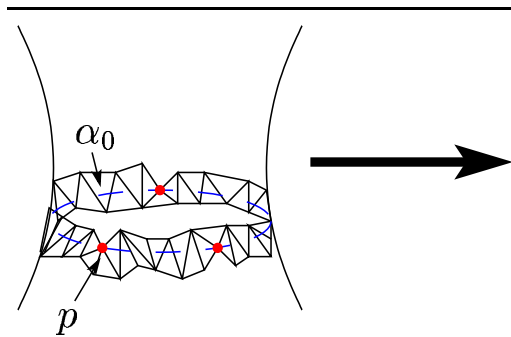

(a)

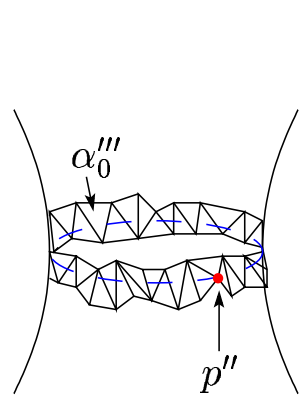

(d)

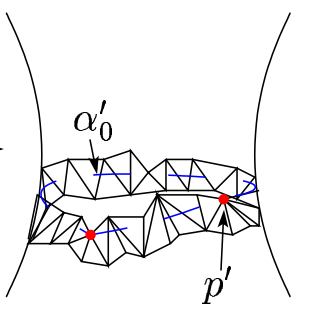

(b)

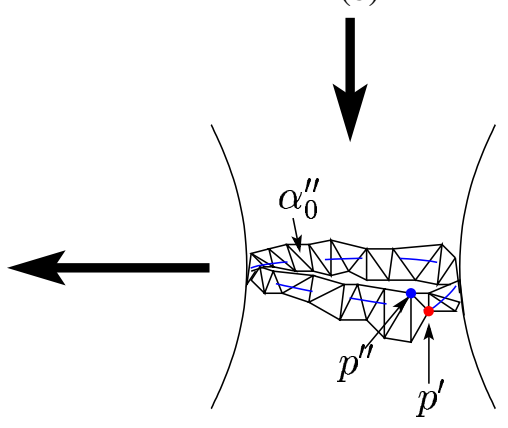

(c)
Figure 4. Overview of the "sliding" algorithm.

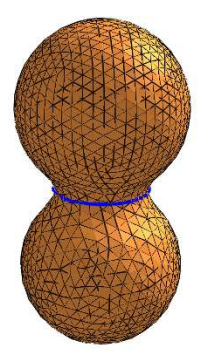

(a)

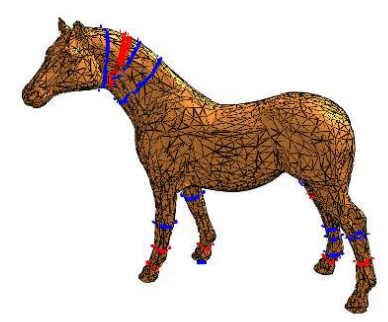

(c)

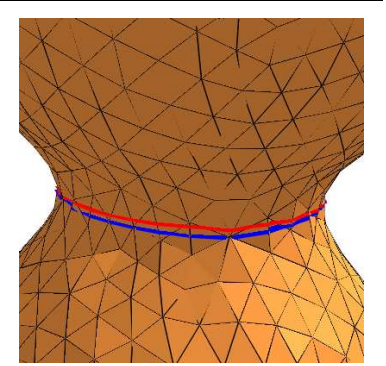

(b)

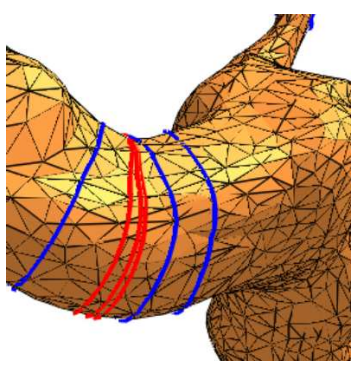

(d)
Figure 5. Constrictions (in red) and the corresponding closed piecewise geodesics (in blue) computed on a dumbbell model $(a, b)$ and a horse model (c,d).

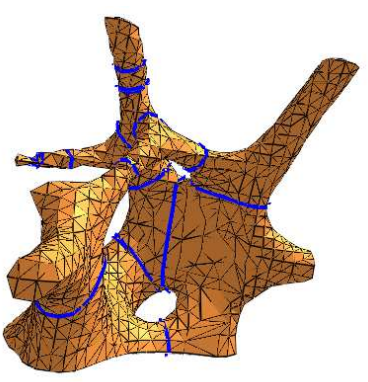

(a)

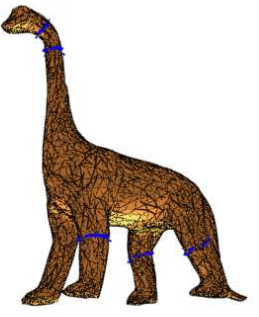

(c)

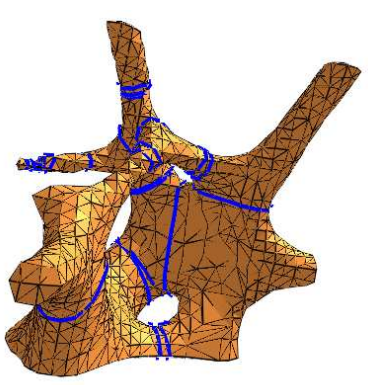

(b)

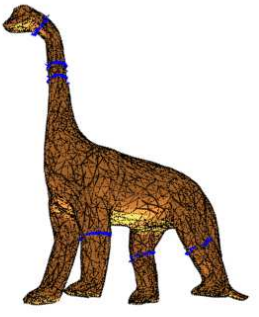

(d)
Figure 6. First type constrictions computed on a surface with genus 3 (a) and on a dinosaur model (c), and the corresponding closed piecewise geodesics $(b, d)$. (a)

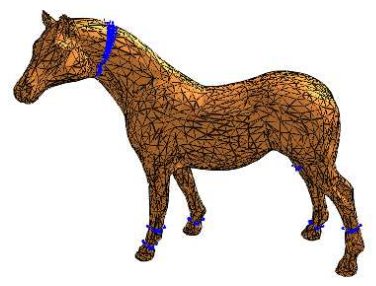

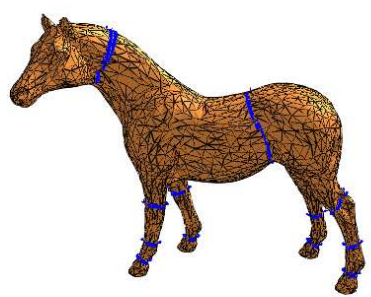

(b)
Figure 7. Constrictions on a horse model computed using Garland and Heckbert's simplification algorithm [3] (a), or selection of the shortest edge at each step (b). 